\title{
MS042.001
}

Microsymposium

\section{Predictive modeling of resonant inelastic $X$-ray scattering with OCEAN}

John Thomas Vinson ${ }^{1}$, Terrence Jach ${ }^{1}$, Matthias Müller ${ }^{2}$, Rainer Unterumsberger ${ }^{2}$, Burkhard Beckhoff ${ }^{2}$

${ }^{1}$ Material Measurement Laboratory, National Institute Of Standards And Technology, Gaithersburg, United States, ${ }^{2}$ PhysikalischTechnische Bundesanstalt, Berlin, Germany

E-mail: john.vinson@nist.gov

Near-edge $x$-ray spectroscopies, including emission and resonant inelastic x-ray scattering (RIXS), are widely used to probe the local electronic and molecular structure of materials. The spectra, however, provide only an indirect measure of the interesting parameters of a system: a transition metal $L$ edge might reveal charge state in a battery cathode whereas the oxygen $\mathrm{K}$ edge can reflect the hydrogen bond network in water. In both cases it is not the spectra themselves that are of interest, but the electronic and structural configurations that give rise to them. Accurate modeling provides an invaluable tool for not only the interpretation of measured results, but also the design of experiments. The OCEAN code simulates near-edge spectra by solving the Bethe-Salpeter equation on top of a density-functional theory foundation, without system-dependent fitting parameters [1]. By contrasting OCEAN results with high-resolution measurements we are able to identify discrepancies that arise from specific effects or physical processes originally neglected in the calculations. We present examples showcasing the importance of correctly accounting for valence-band quasiparticle lifetimes [2], intrinsic disorder including zero-point motion, and phonon scattering when modeling $\mathrm{x}$-ray emission and RIXS.

[1] J. Vinson, et al. (2011). Phys. Rev. B, 83, 115106

[2] J. Vinson, et al. (2016). Phys. Rev. B, 94, 035163

Keywords: RIXS, Bethe-Salpeter equation 